\title{
Velocity and shear stress for an Oldroyd-B fluid within two cylinders
}

\author{
Shin Min Kang ${ }^{1}$, Waqas Nazeer ${ }^{2 *}$, Muhammad Athar ${ }^{2}$, Muhammad Danial Hisham² and \\ Young Chel Kwun ${ }^{3}$
}

"Correspondence:

nazeer.waqas@ue.edu.pk

${ }^{2}$ Division of Science and

Technology, University of Education,

Township, Lahore, Pakistan

Full list of author information is

available at the end of the article

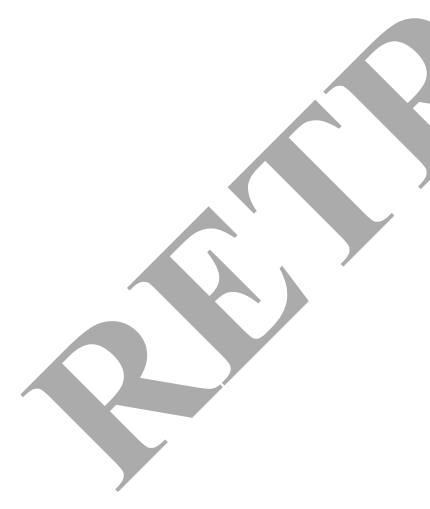

望

Springer

\section{Abstract}

This paper aims to explore the possible solutions for the $m c^{\prime} \mathrm{emt}$ of an Oldroyd-B fluid placed under certain conditions, i.e. the fluid is pres nt h ' in two cylinders, which are coaxial and oscillating within. Having said + the go sing model will be an Oldroyd-B fluid, we wish to achieve our goal of r. Adir. he velocity and shear stress by using some common transformations nely the aplace transformation and the Hankel transformation. The final res' "s, fo the sake of simplicity, will be expressed in the form of generalized G-functic "na urey satisfy all imposed initial and boundary conditions.

Keywords: Oldroyd-B fluid; velocity field; on in, stress; rotational oscillatory flow; Laplace and Hankel transforms

\section{Introduction}

Flow due to an orclitatir. vliy der is one of the most important and interesting problems of motion ne? O. "lating walls. As early as 1886, Stokes [1] established an exact solution to the rotaticrul oscilla ns of an infinite rod immersed in a Newtonian fluid. An extension of this roblem to the rod undergoing both rotational and longitudinal oscillations has been re ed in 2], while the first exact solutions for similar motions of non-Newtonian $\mathrm{fl}^{\mathrm{H}_{\mathrm{C}}}$ are those of Rajagopal [3] and Rajagopal and Bhatnagar [4]. However, all these solut or s a e steady-state solutions to which a transient solution has to be added in order to describe the motion of the fluid for small and large times.

he first closed-form expressions for the starting solutions corresponding to an oscillating motion seem to be those of Erdogan [5] for Newtonian fluids. New exact solutions for the same problem, but presented as a sum of steady-state and transient solutions, have also been established by Corina Fetecau et al. [6]. The extension of these solutions to second grade fluids has been achieved in [7], while the starting solutions for the motion of the same fluids due to longitudinal and torsional oscillations of a circular cylinder have been established in [8]. Recently, starting solutions for oscillating motions of a Maxwell fluid in cylindrical domains have been obtained in [9]. Other interesting results regarding oscillating flows of non-Newtonian fluids have been presented in [10-15].

In this paper, we are interested in the velocity and shear stress for the movement of an Oldroyd-B fluid within two coaxial infinite oscillating cylinders oscillatory motion of a generalized Maxwell fluid between two infinite coaxial circular cylinders, both of them

(c) 2016 Kang et al. This article is distributed under the terms of the Creative Commons Attribution 4.0 International License (http://creativecommons.org/licenses/by/4.0/), which permits unrestricted use, distribution, and reproduction in any medium, provided you give appropriate credit to the original author(s) and the source, provide a link to the Creative Commons license, and indicate if changes were made. 
oscillating around their common axis with given constant angular frequencies $z$. The velocity field and associated tangential stress of the motion are determined by using Laplace and Hankel transforms and are presented by integral and series. It is worthy to point out that the solutions that have been obtained satisfy the governing differential equation and all imposed initial and boundary conditions as well. The solutions correspond to the ordinary Oldroyd-B fluid, performing the same motion.

\subsection{Governing equations of problem}

The movement of the Oldroyd-B fluid is governed by the following mathematical mo del.

$$
\begin{aligned}
& \left(1+\lambda \frac{\partial}{\partial t}\right) \tau(r, t)=\mu\left(1+\lambda_{r} \frac{\partial}{\partial t}\right)\left(\frac{\partial}{\partial r}-\frac{1}{r}\right) w(r, t), \\
& \left(1+\lambda \frac{\partial}{\partial t}\right) \frac{\partial w(r, t)}{\partial t}=v\left(1+\lambda_{r} \frac{\partial}{\partial t}\right)\left(\frac{\partial^{2}}{\partial r^{2}}+\frac{1}{r} \frac{\partial}{\partial r}-\frac{1}{r^{2}}\right) w(r, t) .
\end{aligned}
$$

Here we have labeled the dynamic viscosity as $\mu$, whereas th kin matic viscosity is $v=$ $\frac{\mu}{\rho}$, the constant density of the fluid is presented as $\rho$, the rela tion ume is $\lambda$, and the retardation time is $\lambda_{r}$. We have labeled the velocity $V$ a,$(r, t)$ ar, $u$ the extra-stress $S$ as

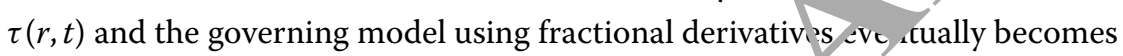

$$
\begin{aligned}
& \left(1+\lambda D_{t}^{\xi}\right) \tau(r, t)=\mu\left(1+\lambda_{r} D_{t}^{\eta}\right)\left(\frac{\partial}{\partial r}-\frac{1}{r}, v(r, t),\right. \\
& \left(1+\lambda D_{t}^{\xi}\right) \frac{\partial w(r, t)}{\partial t}=v\left(1+\lambda_{r} D_{t}^{\eta}\right)\left(\frac{\partial^{2}}{r}, \frac{\partial}{\partial r}-\frac{1}{r^{2}}\right) w(r, t),
\end{aligned}
$$

due to the fractional operator der. d as follows:

$$
\begin{aligned}
D_{t}^{\xi} f(t) & =\frac{1}{\Gamma(1-\xi)} \int \frac{f(\tau)}{(t-\tau)^{\xi}} d \tau \quad \text { when } 0 \leq \xi<1, \\
& =\frac{d}{d t} f(t) \quad \text { en } \xi=1 .
\end{aligned}
$$

We cal to for $\xi$ and $\eta \rightarrow 1$, our model involving fractional derivatives reduces to $t^{1}$ o basic n tel defined earlier due to the fact $D_{t}^{1} f(t)=\frac{d}{d t} f(t)$.

\section{Tht jretical description of the problem}

$\mathrm{Su}$ pose a viscoelastic (Oldroyd-B) fluid is at rest in the annulus of coaxial circular cylincers whose lengths are infinite and having $R_{1}$ and $R_{2}$ radii, respectively, where $R_{1}<R_{2}$. Initially at $t=0$, both the cylinders and the fluid are at rest. At time $t=0^{+}$, the outer cylinder suddenly begin to oscillate around its axis $(r=0)$ with the velocity $Z \sin (z t)$, where $z$ is the constant angular frequency of the outer cylinder and $Z$ is the constant. Owing to the shear, the fluid between the cylinders is gradually moved, its velocity being of the form

$$
\mathbf{V}=\mathbf{V}(r, t)=w(r, t) \mathbf{e}_{\theta},
$$

where $\mathbf{e}_{\theta}$ is the unit vector along $\theta$-direction of the polar coordinate system whose coordinates are $(r, \theta, z)$. 
The constraint of incompressibility is automatically satisfied for this kind of flows. The equation for this motion is

$$
\tau(r, t)=\frac{\mu\left(1+\lambda_{r} D_{t}^{\eta}\right)}{\left(1+\lambda D_{t}^{\xi}\right)}\left(\frac{\partial}{\partial r}-\frac{1}{r}\right) w(r, t)
$$

where $\tau(r, t)=S_{r \theta}(r, t)$ is the only non-zero shear stress. When the pressure gradient and the body forces in the axial direction are absent, the following equation is obtained by the balance of the linear momentum:

$$
\rho \frac{\partial w(r, t)}{\partial t}=\left(\frac{\partial}{\partial r}+\frac{2}{r}\right) \tau(r, t)
$$

where the constant density of the fluid is $\rho$.

In this paper, we have determined the velocity and the shear stress wh the inner cylinder is fixed and the outer cylinder is moving. The initial and bo - yary con cons, when the inner cylinder is fixed and the outer cylinder moves grad lly s some

$$
\begin{aligned}
& w(r, 0)=0 ; \quad r \in\left[R_{1}, R_{2}\right], \\
& w\left(R_{1}, t\right)=0, \quad w\left(R_{2}, t\right)=Z \sin (z t) .
\end{aligned}
$$

Also

$$
\bar{w}\left(R_{1}, s\right)=0, \quad \bar{w}\left(R_{2}, s\right)=\frac{Z z}{z^{2}+s^{-}}
$$

Two transformations, name, the place and the Hankel transformations, can be applied to the problem to solve $:$.

\section{Calculation of the incity field}

Let us apply Lap 'mansformation to equation (4) to obtain the following ODE:

$$
\text { (. } \lambda s^{\xi} \bar{\nu}_{\bar{\nu}}(r, s)=v\left(1+\lambda_{r} s^{\eta}\right)\left(\frac{\partial^{2}}{\partial r^{2}}+\frac{1}{r} \frac{\partial}{\partial r}-\frac{1}{r^{2}}\right) \bar{w}(r, s) \text {, }
$$

Wh. ' ' $s$ ' is the parameter of the Laplace transform, or

$$
\frac{s+\lambda s^{\xi+1}}{\nu\left(1+\lambda r^{\eta}\right)} \bar{w}(r, s)=\left(\frac{\partial^{2}}{\partial r^{2}}+\frac{1}{r} \frac{\partial}{\partial r}-\frac{1}{r^{2}}\right) \bar{w}(r, s) .
$$

Multiplying both sides of above equation by $r B_{1}\left(r, r_{n}\right)$ and integrating with respect to ' $r$ ' from $R_{1}$ to $R_{2}$, where $B_{1}\left(r, r_{n}\right)=J_{1}\left(r r_{n}\right) Y_{1}\left(R_{2} r_{n}\right)-J_{1}\left(R_{2} r_{n}\right) Y_{1}\left(r r_{n}\right)$, and $r_{n}$ are the positive roots of the equation $B_{1}\left(R_{1} r_{n}\right)=0$.

Hence our last equation becomes

$$
\begin{aligned}
& \frac{s+\lambda s^{\xi+1}}{v\left(1+\lambda_{r} s^{\eta}\right)} \int_{R_{1}}^{R_{2}} r B_{1}\left(r r_{n}\right) \bar{w}(r, s) d r \\
& =\int_{R_{1}}^{R_{2}} r\left(\frac{\partial^{2}}{\partial r^{2}}+\frac{1}{r} \frac{\partial}{\partial r}-\frac{1}{r^{2}}\right) B_{1}\left(r r_{n}\right) \bar{w}(r, s) d r .
\end{aligned}
$$


Also we define the Hankel transform of $\bar{w}(r, s)$ as

$$
\bar{W}_{H}\left(r_{n}, s\right)=\int_{R_{1}}^{R_{2}} r \bar{w}(r, s) B_{1}\left(r r_{n}\right) d r .
$$

Consider right hand side of the above equation (14), and solving it for simplification purposes, we get

$$
\int_{R_{1}}^{R_{2}} r\left(\frac{\partial^{2}}{\partial r^{2}}+\frac{1}{r} \frac{\partial}{\partial r}-\frac{1}{r^{2}}\right) B_{1}\left(r r_{n}\right) \bar{w}(r, s) d r=\frac{2 Z z}{\pi\left(z^{2}+s^{2}\right)}-r_{n}^{2} \bar{W}_{H}\left(r_{n}, s\right) .
$$

Again, from equation (14), we can deduce that

$$
\frac{s+\lambda s^{\xi+1}}{v\left(1+\lambda_{r} s^{\eta}\right)} \bar{W}_{H}\left(r_{n}, s\right)=\frac{2 Z z}{\pi\left(z^{2}+s^{2}\right)}-r_{n}^{2} \bar{W}_{H}\left(r_{n}, s\right)
$$

Again simplifying the above equation for $\bar{W}_{H}\left(r_{n}, s\right)$, we get

$$
\bar{W}_{H}\left(r_{n}, s\right)=\frac{2 Z z}{\pi\left(z^{2}+s^{2}\right)} \frac{v\left(1+\lambda_{r} s^{\eta}\right)}{s+\lambda s^{\xi+1}+v r_{n}^{2}+v r_{n}^{2} \lambda_{r} s^{\eta}} .
$$

More simplification gives us

$$
\bar{W}_{H}\left(r_{n}, s\right)=\frac{2 Z z}{r_{n}^{2} \pi\left(z^{2}+s^{2}\right)}-\frac{7_{n}\left(s+\frac{\xi+1}{r_{n}^{2}\left(\pi\left(z^{2}, \alpha^{2}\right)\right)}\left(s s^{\xi}\right.\right.}{\left.+v r_{n}^{2}+v r_{n}^{2} \lambda_{r} s^{\eta}\right)} .
$$

Or equivalently, we write $\bar{W}_{H}\left(r_{n},{ }_{0}=r_{n}, r_{n},\right)-\bar{W}_{2 H}\left(r_{n}, s\right)$, where

$$
\bar{W}_{1 H}\left(r_{n}, s\right)=\frac{2 Z z}{r_{n}^{2} \pi\left(z^{2}+s^{2}\right)}
$$

and

$$
\bar{W}_{2 H}\left(r_{n}, s\right)=\frac{2 Z z\left(s+\lambda s^{\xi+1}\right)}{\left(\pi\left(z^{2}+s^{2}\right)\right)\left(s+\lambda s^{\xi+1}+v r_{n}^{2}+v r_{n}^{2} \lambda_{r} s^{\eta}\right)}
$$

B fort sroceed, let us define the inverse Hankel transform

$$
(r, s)=\frac{Z z}{\left(s^{2}+z^{2}\right)} \frac{R_{2}\left(r^{2}-R_{1}^{2}\right)}{\left(R_{2}^{2}-R_{1}^{2}\right) r}
$$

and

$$
\bar{w}_{2}(r, s)=\frac{\pi^{2}}{2} \sum_{n=1}^{\infty} \frac{r_{n}^{2} J_{b 1}^{2}\left(R_{1} r_{n}\right) B_{1}\left(r r_{n}\right)}{J_{b 1}^{2}\left(R_{1} r_{n}\right)-J_{b 1}^{2}\left(R_{2} r_{n}\right)} \bar{W}_{2 H}\left(r_{n}, s\right) .
$$

This leads us to

$$
\begin{aligned}
\bar{w}(r, s)= & \frac{Z z}{\left(z^{2}+s^{2}\right)} \frac{R_{2}\left(r^{2}-R_{1}^{2}\right)}{r\left(R_{2}^{2}-R_{1}^{2}\right)}-\frac{\pi^{2}}{2} \sum_{n=1}^{\infty} \frac{r_{n}^{2} J_{b 1}^{2}\left(R_{1} r_{n}\right) B_{1}\left(r r_{n}\right)}{J_{b 1}^{2}\left(R_{1} r_{n}\right)-J_{b 1}^{2}\left(R_{2} r_{n}\right)} \\
& \times\left[\frac{2 Z z\left(s+\lambda s^{\xi+1}\right)}{r_{n}^{2}\left(\pi\left(z^{2}+s^{2}\right)\right)\left(s+\lambda s^{\xi+1}+v r_{n}^{2}+v r_{n}^{2} \lambda_{r} s^{\eta}\right)}\right]
\end{aligned}
$$


or equivalently

$$
\begin{aligned}
\bar{w}(r, s)= & \frac{Z z}{\left(z^{2}+s^{2}\right)} \frac{R_{2}\left(r^{2}-R_{1}^{2}\right)}{r\left(R_{2}^{2}-R_{1}^{2}\right)}-\pi \sum_{n=1}^{\infty} \frac{J_{b 1}^{2}\left(R_{1} r_{n}\right) B_{1}\left(r r_{n}\right)}{J_{b 1}^{2}\left(R_{1} r_{n}\right)-J_{b 1}^{2}\left(R_{2} r_{n}\right)} \\
& \times\left[\frac{Z z\left(s+\lambda s^{\xi+1}\right)}{\left(z^{2}+s^{2}\right)\left(s+\lambda s^{\xi+1}+\nu r_{n}^{2}+\nu r_{n}^{2} \lambda_{r} s^{\eta}\right)}\right] .
\end{aligned}
$$

Equivalently,

$$
\frac{1}{s+\lambda s^{\xi+1}+v r_{n}^{2}+v r_{n}^{2} \lambda_{r} s^{\eta}}=\frac{1}{\lambda} \sum_{k=0}^{\infty} \sum_{m=0}^{k} \lambda_{r}^{m}\left(\frac{-v r_{n}^{2}}{\lambda}\right)^{k} \frac{s^{\eta m-k-1}}{\left(s^{\xi}+\frac{1}{\lambda}\right)^{k+1}},
$$

and consequently

$$
\begin{aligned}
\bar{w}(r, s)= & \frac{Z z}{\left(z^{2}+s^{2}\right)} \frac{R_{2}\left(r^{2}-R_{1}^{2}\right)}{r\left(R_{2}^{2}-R_{1}^{2}\right)}-\pi \sum_{n=1}^{\infty} \frac{J_{b 1}^{2}\left(R_{1} r_{n}\right) B_{1}\left(r r_{n}\right)}{J_{b 1}^{2}\left(R_{1} r_{n}\right)-J_{b 1}^{2}\left(R_{2} r_{n}\right)} \\
& \times\left[\frac{Z z\left(s+\lambda s^{\xi+1}\right)}{\left(z^{2}+s^{2}\right)} \frac{1}{\lambda} \sum_{k=0}^{\infty} \sum_{m=0}^{k} \lambda_{r}^{m}\left(\frac{-v r_{n}^{2}}{\lambda}\right)^{k} \frac{s^{\prime}}{\left(s^{\xi}+\frac{7}{\lambda}\right)^{k+2}}\right]
\end{aligned}
$$

or

$$
\begin{aligned}
\bar{w}(r, s)= & \frac{Z z}{\left(z^{2}+s^{2}\right)} \frac{R_{2}\left(r^{2}-R_{1}^{2}\right)}{r\left(R_{2}^{2}-R_{1}^{2}\right)} \sum_{n=1}^{\infty} \frac{J_{b}^{2}}{T_{b_{1}}^{2}\left(R_{1} r_{n}\right)-J_{b 1}^{2}\left(R_{2} r_{n}\right)} \\
& \times \frac{1}{\lambda} \sum_{k=0}^{\infty} \sum_{=0}^{k} \lambda_{r}^{\eta}\left(\frac { - \nu _ { n } } { \lambda } r ^ { k } \left[\left(\frac{Z z}{\left(z^{2}+s^{2}\right)}\right)\left(\frac{s^{\eta m-k}}{\left(s^{\xi}+\frac{1}{\lambda}\right)^{k+1}}\right)\right.\right. \\
& \left.+\left(\frac{\lambda}{\left.-2+s^{2}\right)}\right)\left(\frac{s^{\eta m-k+\xi}}{\left(s^{\xi}+\frac{1}{\lambda}\right)^{k+1}}\right)\right] .
\end{aligned}
$$

Taking he 1 place irverse using the convolution theorem and the identity

$$
G_{a, b, c}(d, t)=L^{-1}\left(\frac{s^{b}}{\left(s^{a}-d\right)^{c}},\right)
$$

$\operatorname{Rf}^{\prime}(a c-b)>0, \operatorname{Re}(s)>0,\left|\frac{d}{s^{a}}\right|>0$, we get the shape of the above equation as

$$
\begin{aligned}
w(r, t)= & \frac{R_{2}\left(r^{2}-R_{1}^{2}\right)(Z \sin z t)}{r\left(R_{2}^{2}-R_{1}^{2}\right)}-\frac{Z \pi}{\lambda} \sum_{n=1}^{\infty} \frac{J_{b 1}^{2}\left(R_{1} r_{n}\right) B_{1}\left(r r_{n}\right)}{J_{b 1}^{2}\left(R_{1} r_{n}\right)-J_{b 1}^{2}\left(R_{2} r_{n}\right)} \\
& \times \sum_{k=0}^{\infty} \sum_{m=0}^{k} \lambda_{r}^{m}\left(\frac{-v r_{n}^{2}}{\lambda}\right)^{k}\left[\int_{0}^{t} \sin z(t-\tau) G_{\xi, \eta m-k, k+1}\left(\frac{-1}{\lambda}, \tau\right) d \tau\right. \\
& \left.+\lambda \int_{0}^{t} \sin z(t-\tau) G_{\xi, \eta m-k+\xi, k+1}\left(\frac{-1}{\lambda}, \tau\right) d \tau\right]
\end{aligned}
$$

which is the required velocity field. 


\subsection{Calculation of shear stress}

Considering equation (3) and solving it for $\tau(r, t)$, we get

$$
\tau(r, t)=\frac{\mu\left(1+\lambda_{r} D_{t}^{\eta}\right)}{\left(1+\lambda D_{t}^{\xi}\right)}\left(\frac{\partial}{\partial r}-\frac{1}{r}\right) w(r, t)
$$

taking the Laplace transform on both sides, we get

$$
\bar{\tau}(r, s)=\frac{\mu\left(1+\lambda_{r} s^{\eta}\right)}{\left(1+\lambda s^{\xi}\right)}\left(\frac{\partial}{\partial r}-\frac{1}{r}\right) \bar{w}(r, s),
$$

obtaining the value of $\bar{w}(r, s)$ from equation (25) and putting it in the above qua n; we need to first calculate $\left(\frac{\partial}{\partial r}-\frac{1}{r}\right) \bar{w}(r, s)$,

$$
\begin{aligned}
& \left(\frac{\partial}{\partial r}-\frac{1}{r}\right) \bar{w}(r, s)=\frac{2 Z z}{\left(z^{2}+s^{2}\right)} \frac{R_{2} R_{1}^{2}}{r^{2}\left(R_{2}^{2}-R_{1}^{2}\right)}+\frac{\pi}{\lambda} \sum_{n=0}^{\infty} \frac{J_{1}^{2}\left(R_{1} r_{n}\right)\left(2 B_{1}\left(r r_{n}\right)-r r_{n}\right.}{r\left(J_{1}^{2}\left(P_{1} r_{n}, J_{n}\right)\right)} \\
& \times \sum_{k=0}^{\infty} \sum_{m=0}^{k} \lambda_{r}^{m}\left(\frac{-v r_{n}^{2}}{\lambda}\right)^{k}\left[\left(\frac{Z z}{\left(z^{2}+s^{2}\right.}\right)\left(\frac{s}{\left(n \xi+\frac{1}{\lambda}\right.} \frac{-k}{k+1}\right)\right. \\
& \left.+\lambda\left(\frac{Z z}{\left(z^{2}+s^{2}\right)}\right)\left(\frac{s^{\eta m-k+\xi}}{\left(s^{\xi}+\frac{1}{\lambda}\right)^{\wedge}}\right)\right]
\end{aligned}
$$

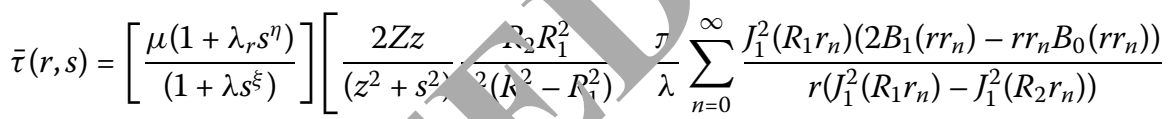

$$
\begin{aligned}
& \times \sum_{k=0}^{\infty} \sum_{m=0}^{k} \lambda_{r}^{m}\left(-\frac{\lambda}{\lambda}\right) \sqrt{Z^{k}} \frac{Z_{k}}{\left.z^{2}+s^{2}\right)}\left(\frac{s^{\eta m-k}}{\left(s^{\xi}+\frac{1}{\lambda}\right)^{k+1}}\right) \\
& \left.+\lambda\left(\frac{Z}{\left(z^{2}+\right.}\right)\left(\frac{s)^{\eta m-k+\xi}}{\left.s s^{\xi}+\frac{1}{\lambda}\right)^{k+1}}\right)\right],
\end{aligned}
$$

or

$$
\begin{aligned}
\overline{\bar{c}}\left(r, \mathrm{~d}, \frac{\mu}{\left(1+\lambda s^{\xi}\right)}\right]\left[\frac{2 Z z}{\left(z^{2}+s^{2}\right)} \frac{R_{2} R_{1}^{2}}{r^{2}\left(R_{2}^{2}-R_{1}^{2}\right)}+\frac{\pi}{\lambda} \sum_{n=0}^{\infty} \frac{J_{1}^{2}\left(R_{1} r_{n}\right)\left(2 B_{1}\left(r r_{n}\right)-r r_{n} B_{0}\left(r r_{n}\right)\right)}{r\left(J_{1}^{2}\left(R_{1} r_{n}\right)-J_{1}^{2}\left(R_{2} r_{n}\right)\right)}\right. \\
\quad \times \sum_{k=0}^{\infty} \sum_{m=0}^{k} \lambda_{r}^{m}\left(\frac{-v r_{n}^{2}}{\lambda}\right)^{k}\left[\frac{Z z}{\left(z^{2}+s^{2}\right)}\left(\frac{s^{\eta m-k}}{\left(s^{\xi}+\frac{1}{\lambda}\right)^{k+1}}\right)\right. \\
\left.\left.+\lambda\left(\frac{Z z}{\left(z^{2}+s^{2}\right)}\right)\left(\frac{s^{\eta m-k+\xi}}{\left(s^{\xi}+\frac{1}{\lambda}\right)^{k+1}}\right)\right]\right] \\
+\left[\frac{\mu \lambda_{r} s^{\eta}}{\left(1+\lambda s^{\xi}\right)}\right]\left[\frac{2 Z z}{\left(z^{2}+s^{2}\right)} \frac{R_{2} R_{1}^{2}}{r^{2}\left(R_{2}^{2}-R_{1}^{2}\right)}+\frac{\pi}{\lambda} \sum_{n=0}^{\infty} \frac{J_{1}^{2}\left(R_{1} r_{n}\right)\left(2 B_{1}\left(r r_{n}\right)-r r_{n} B_{0}\left(r r_{n}\right)\right)}{r\left(J_{1}^{2}\left(R_{1} r_{n}\right)-J_{1}^{2}\left(R_{2} r_{n}\right)\right)}\right. \\
+\sum_{k=0}^{\infty} \sum_{m=0}^{k} \lambda_{r}^{m}\left(\frac{-v r_{n}^{2}}{\lambda}\right)^{k}\left[\frac{Z z}{\left(z^{2}+s^{2}\right)}\left(\frac{s^{\eta m-k}}{\left(s^{\xi}+\frac{1}{\lambda}\right)^{k+1}}\right)\right. \\
\left.\left.+\lambda\left(\frac{Z z}{\left(z^{2}+s^{2}\right)}\right)\left(\frac{s^{\eta m-k+\xi}}{\left(s^{\xi}+\frac{1}{\lambda}\right)^{k+1}}\right)\right]\right]
\end{aligned}
$$


Equivalently

$$
\begin{aligned}
\bar{\tau}(r, s)= & \left.\frac{\mu}{\lambda}\right]\left[\frac{2 Z z}{\left(z^{2}+s^{2}\right)} \frac{R_{2} R_{1}^{2}}{r^{2}\left(R_{2}^{2}-R_{1}^{2}\right)} \frac{s^{0}}{s^{\xi}+\frac{1}{\lambda}}+\frac{\pi}{\lambda} \sum_{n=0}^{\infty} \frac{J_{1}^{2}\left(R_{1} r_{n}\right)\left(2 B_{1}\left(r r_{n}\right)-r r_{n} B_{0}\left(r r_{n}\right)\right)}{r\left(J_{1}^{2}\left(R_{1} r_{n}\right)-J_{1}^{2}\left(R_{2} r_{n}\right)\right)}\right. \\
& \times \sum_{k=0}^{\infty} \sum_{m=0}^{k} \lambda_{r}^{m}\left(\frac{-v r_{n}^{2}}{\lambda}\right)^{k}\left[\frac{Z z}{\left(z^{2}+s^{2}\right)}\left(\frac{s^{\eta m-k}}{\left(s^{\xi}+\frac{1}{\lambda}\right)^{k+1}}\right)\right. \\
& \left.\left.+\lambda\left(\frac{Z z}{\left(z^{2}+s^{2}\right)}\right)\left(\frac{s^{\eta m-k+\xi}}{\left(s^{\xi}+\frac{1}{\lambda}\right)^{k+1}}\right)\right]\right] \\
& +\left[\frac{\mu \lambda r}{\lambda}\right]\left[\frac{2 Z z}{\left(z^{2}+s^{2}\right)} \frac{R_{2} R_{1}^{2}}{r^{2}\left(R_{2}^{2}-R_{1}^{2}\right)}+\frac{\pi}{\lambda} \sum_{n=0}^{\infty} \frac{J_{1}^{2}\left(R_{1} r_{n}\right)\left(2 B_{1}\left(r r_{n}\right)-r r_{n} B_{0}(r r)\right.}{r\left(J_{1}^{2}\left(R_{1} r_{n}\right)-J_{1}^{2}\left(R_{2} r_{n}\right.\right.}\right. \\
& \times \sum_{k=0}^{\infty} \sum_{m=0}^{k} \lambda_{r}^{m}\left(\frac{-v r_{n}^{2}}{\lambda}\right)^{k}\left[\frac{Z z}{\left(z^{2}+s^{2}\right)}\left(\frac{s^{\eta m-k+\eta}}{\left(s^{\xi}+\frac{1}{\lambda}\right)^{k+2}}\right)\right. \\
& \left.\left.+\lambda\left(\frac{Z z}{\left(z^{2}+s^{2}\right)}\right)\left(\frac{s^{\eta m-k+\xi+\eta}}{\left(s^{\xi}+\frac{1}{\lambda}\right)^{k+2}}\right)\right]\right]
\end{aligned}
$$

Taking the Laplace inverse, using the convolution theoren, and the following identity:

$$
G_{a, b, c}(d, t)=L^{-1}\left(\frac{s^{b}}{\left(s^{a}-d\right)^{c}}\right)
$$

$\operatorname{Re}(a c-b)>0, \operatorname{Re}(s)>0,\left|\frac{d}{s^{a}}\right|>c$

$$
\begin{aligned}
\tau(r, t)= & \left.\frac{\mu}{\lambda}\right]\left[\frac{27 R_{2} R_{1}^{2}}{r^{2}} \int_{0}^{t} \sin z(t-\tau) G_{0, \xi, 1}\left(\frac{-1}{\lambda}, \tau\right) d \tau\right. \\
& +\frac{\left.R_{1}^{2}\right)}{r} \sum_{m=0}^{\infty} \lambda_{r}^{m}\left(\frac{-v r_{n}^{2}}{\lambda}\right)^{k}\left[\int_{0}^{t} \sin z(t-\tau) G_{\eta m-k, \xi, k+2}\left(\frac{-1}{\lambda}, \tau\right) d \tau\right. \\
& \left.\left.+\lambda \int_{0}^{t} \sin z(t-\tau) G_{\eta m-k+\xi, \xi, k+2}\left(\frac{-1}{\lambda}, \tau\right) d \tau\right]\right] \\
& +\left[\frac{\mu \lambda_{r}}{\lambda}\right]\left[\frac{\left.\left.2 Z R_{1} r_{n}\right)-J_{1}^{2}\left(R_{2} r_{n}\right)\right)}{r^{2}\left(R_{2}^{2}-R_{1}^{2}\right)} \int_{0}^{t} \sin z(t-\tau) G_{\eta, \xi, 1}\left(\frac{-1}{\lambda}, \tau\right) d \tau\right. \\
& +\frac{Z \pi}{\lambda} \sum_{n=0}^{\infty} \frac{J_{1}^{2}\left(R_{1} r_{n}\right)\left(2 B_{1}\left(r r_{n}\right)-r r_{n} B_{0}\left(r r_{n}\right)\right)}{r\left(J_{1}^{2}\left(R_{1} r_{n}\right)-J_{1}^{2}\left(R_{2} r_{n}\right)\right)} \\
& \times \sum_{k=0}^{\infty} \sum_{m=0}^{k} \lambda_{r}^{m}\left(\frac{-v r_{n}^{2}}{\lambda}\right)^{k}\left[\int_{0}^{t} \sin z(t-\tau) G_{\eta m-k+\eta, \xi, k+2}\left(\frac{-1}{\lambda}, \tau\right) d \tau\right. \\
& \left.\left.+\lambda \int_{0}^{t} \sin z(t-\tau) G_{\eta m-k+\xi+\eta, \xi, k+2}\left(\frac{-1}{\lambda}, \tau\right) d \tau\right]\right] .
\end{aligned}
$$




\section{Particularization of the above results}

The above results are of a general nature and the imposition of certain limits/conditions may bring these to particular fluids.

\subsection{Ordinary Oldroyd-B fluid}

The velocity field and shear stress of the movement of an ordinary Oldroyd-B fluid can be deduced imposing $\xi, \eta \rightarrow 1$ on the obtained results:

$$
\begin{aligned}
w(r, t)= & \frac{R_{2}\left(r^{2}-R_{1}^{2}\right)(Z \sin z t)}{r\left(R_{2}^{2}-R_{1}^{2}\right)} \\
& -\frac{Z \pi}{\lambda} \sum_{n=1}^{\infty} \frac{J_{b 1}^{2}\left(R_{1} r_{n}\right) B_{1}\left(r r_{n}\right)}{J_{b 1}^{2}\left(R_{1} r_{n}\right)-J_{b 1}^{2}\left(R_{2} r_{n}\right)} \\
& \times \sum_{k=0}^{\infty} \sum_{m=0}^{k} \lambda_{r}^{m}\left(\frac{-v r_{n}^{2}}{\lambda}\right)^{k}\left[\int_{0}^{t} \sin z(t-\tau) G_{1, m-k, k+1}\left(-\frac{1}{\lambda}\right)\right) d \tau \\
& \left.+\lambda \int_{0}^{t} \sin z(t-\tau) G_{1, m-k+1, k+1}\left(\frac{-1}{\lambda}, \tau\right) d \tau\right]
\end{aligned}
$$

and the associated shear stress will take the from

$$
\begin{aligned}
& \tau(r, t)=\left[\frac{\mu}{\lambda}\right]\left[\frac{2 Z R_{2} R_{1}^{2}}{r^{2}\left(R_{2}^{2}-R_{1}^{2}\right)} \int_{0}^{t} S ;(t-\tau) G_{0, \eta, 1}\left(\frac{-1}{\lambda}, \tau\right) d \tau\right. \\
& +\frac{Z \pi}{\lambda} \sum_{n=0}^{\infty} \frac{J_{1}^{2}(R}{r\left(J _ { 1 } ^ { 2 } \left(R_{1}\right.\right.} \frac{\left.\mathrm{Q}_{1}\left(r r_{n}\right)-r r_{n} B_{0}\left(r r_{n}\right)\right)}{\left.-J_{1}^{2}\left(R_{2} r_{n}\right)\right)} \\
& \times \sum_{m=0}^{\infty} \sum_{\lambda}^{k}\left(\frac{-v \eta^{2}}{\lambda}\right)^{k}\left[\int_{0}^{t} \sin z(t-\tau) G_{m-k, 1, k+2}\left(\frac{-1}{\lambda}, \tau\right) d \tau\right. \\
& \left.\left.+\lambda \int_{0}^{t} \sin z(t-\tau) G_{m-k+1,1, k+2}\left(\frac{-1}{\lambda}, \tau\right) d \tau\right]\right] \\
& +\left[\frac{\mu \lambda_{r}}{\lambda}\right]\left[\frac{2 Z R_{2} R_{1}^{2}}{r^{2}\left(R_{2}^{2}-R_{1}^{2}\right)} \int_{0}^{t} \sin z(t-\tau) G_{1,1,1}\left(\frac{-1}{\lambda}, \tau\right) d \tau\right. \\
& +\frac{Z \pi}{\lambda} \sum_{n=0}^{\infty} \frac{J_{1}^{2}\left(R_{1} r_{n}\right)\left(2 B_{1}\left(r r_{n}\right)-r r_{n} B_{0}\left(r r_{n}\right)\right)}{r\left(J_{1}^{2}\left(R_{1} r_{n}\right)-J_{1}^{2}\left(R_{2} r_{n}\right)\right)} \\
& \times \sum_{k=0}^{\infty} \sum_{m=0}^{k} \lambda_{r}^{m}\left(\frac{-v r_{n}^{2}}{\lambda}\right)^{k}\left[\int_{0}^{t} \sin z(t-\tau) G_{m-k+1,1, k+2}\left(\frac{-1}{\lambda}, \tau\right) d \tau\right. \\
& \left.\left.+\lambda \int_{0}^{t} \sin z(t-\tau) G_{m-k+2,1, k+2}\left(\frac{-1}{\lambda}, \tau\right) d \tau\right]\right] .
\end{aligned}
$$

\subsection{Ordinary Maxwell fluid}

If $\xi \rightarrow 1, \lambda_{r} \rightarrow 0$ in the already found results for the velocity and shear stress then the resultants will govern the movement of an ordinary Maxwell fluid under the same cir- 
cumstances. We have

$$
\begin{aligned}
w(r, t)= & \frac{R_{2}\left(r^{2}-R_{1}^{2}\right)(Z \sin z t)}{r\left(R_{2}^{2}-R_{1}^{2}\right)} \\
& -\frac{Z \pi}{\lambda} \sum_{n=1}^{\infty} \frac{J_{b 1}^{2}\left(R_{1} r_{n}\right) B_{1}\left(r r_{n}\right)}{J_{b 1}^{2}\left(R_{1} r_{n}\right)-J_{b 1}^{2}\left(R_{2} r_{n}\right)} \\
& \times \sum_{k=0}^{\infty}\left(\frac{-v r_{n}^{2}}{\lambda}\right)^{k}\left[\int_{0}^{t} \sin z(t-\tau) G_{1,-k, k+1}\left(\frac{-1}{\lambda}, \tau\right) d \tau\right. \\
& \left.+\lambda \int_{0}^{t} \sin z(t-\tau) G_{1,-k+1, k+1}\left(\frac{-1}{\lambda}, \tau\right) d \tau\right]
\end{aligned}
$$

and

$$
\begin{aligned}
& \tau(r, t)=\left[\frac{\mu}{\lambda}\right]\left[\frac{2 Z R_{2} R_{1}^{2}}{r^{2}\left(R_{2}^{2}-R_{1}^{2}\right)} \int_{0}^{t} \sin z(t-\tau) G_{0,1,1}\left(\frac{-1}{\lambda}, \tau\right) d \tau\right. \\
& +\frac{Z \pi}{\lambda} \sum_{n=0}^{\infty} \frac{J_{1}^{2}\left(R_{1} r_{n}\right)\left(2 B_{1}\left(r r_{n}\right)-r r_{n} B_{0}\left(r r_{n}\right)\right)}{r\left(J_{1}^{2}\left(R_{1} r_{n}\right)-J_{1}^{2}\left(R_{2} r_{n}\right)\right)} \\
& \times \sum_{k=0}^{\infty}\left(\frac{-v r_{n}^{2}}{\lambda}\right)^{k}\left[\int_{0}^{t} \sin z(t-\tau) G \ldots+2\left(\frac{-1}{\lambda}, t\right) d \tau\right. \\
& \left.+\lambda \int_{0}^{t} \sin z(t-\tau) G_{-k+1}, k+2\left(\frac{-1}{\lambda}, \tau, \tau\right]\right] \\
& +\frac{Z \pi}{\lambda} \sum_{n=0}^{\infty} \frac{J_{1}^{2}\left(R_{1} r\right.}{V_{1}^{2}\left(B_{1}\left(r r_{n}\right)\right.} \frac{\left.r_{n} B_{0}\left(r r_{n}\right)\right)}{\left.J_{1}^{2}\left(R_{2} r_{n}\right)\right)} \\
& \times \sum_{k=0}^{\infty}\left(-r_{n}^{2}\right)^{k}\left[\int_{0}^{t} \sin z(t-\tau) G_{-k+\eta, 1, k+2}\left(\frac{-1}{\lambda}, \tau\right) d \tau\right. \\
& \left.+\lambda \int_{1} \sin ,-(t-\tau) G_{-k+1+\eta, 1, k+2}\left(\frac{-1}{\lambda}, \tau\right) d \tau\right] .
\end{aligned}
$$

5 ronc 10.1

rabove deavors were to develop a formula for the calculation of exact solutions for the ocity field and the shear stress of the motion (flow) of an Oldroyd-B fluid present betwe $n$ two rotationally oscillating cylinders of infinite lengths. The use of fractional de ivatives and the commonly known transformations, i.e. the Laplace and the Hankel cransformations, has made the approach more accessible. The central notion depicts the phenomenon that a viscoelastic (Oldroyd-B) fluid will react under certain conditions and that can we control such flow. At first stage the inner cylinder was supposed to be at rest, i.e. fixed, whereas the movement was produced by the outer cylinder. At the second stage, we analyzed the flow of the fluid produced by the movement of the inner cylinder while considering the outer cylinder at rest or fixed. The obtained solutions satisfy the governing equations and all imposed initial and boundary conditions. The solutions, obtained by means of Laplace and Hankel transforms, are presented in integral and series forms in terms of the generalized G-function. In the end these general solutions have been particularized for 'ordinary Oldroyd-B fluids' and for 'ordinary Maxwell fluids'. 


\section{Appendix}

The following are some expressions used in the text:

(A1) The finite Hankel transform of the function

$$
a(r)=\frac{C_{1} R_{1}\left(R_{2}^{2}-r^{2}\right)+C_{2} R_{2}\left(r^{2}-R_{1}^{2}\right)}{\left(R_{2}^{2}-R_{1}^{2}\right) r}
$$

satisfying $a\left(R_{1}\right)=C_{1}$ and $a\left(R_{2}\right)=C_{2}$ is

$$
a_{n}(r)=\int_{R_{1}}^{R_{2}} r a(r) B_{1}\left(r r_{n}\right) d r=\frac{2 C_{2}}{\pi r_{n}^{2}}-\frac{2 C_{1}}{\pi r_{n}^{2}} \frac{J_{1}\left(R_{2} r_{n}\right)}{J_{1}\left(R_{1} r_{n}\right)} .
$$

(A2) If $f(t)=L^{-1}\{\bar{f}(q)\}$ and $g(t)=L^{-1}\{\bar{g}(q)\}$, then

$$
\begin{aligned}
L^{-1}\{\bar{f}(q) \bar{g}(q)\} & =(f * g)(t) \\
& =\int_{0}^{t} f(t-\tau) g(\tau) d \tau \\
& =\int_{0}^{t} f(t) g(t-\tau) d \tau .
\end{aligned}
$$

(A3)

$$
\sum_{k=0}^{\infty}\left(-v r_{n}^{2}\right)^{k} G_{0,-1-k, k+1}\left(-c r_{n}^{2}, t\right)=\frac{}{1+\alpha r_{n}^{2}} \exp \left(\frac{-v r_{n}^{2} t}{1+\alpha r_{n}^{2}}\right) .
$$

Competing interests

The authors declare that they ha ve no competing, nterests.

\section{Authors' contributions}

All authors contribute equally in rese, ch.

\section{Author details}

'Department of Mathen at; cs ar, a RINS, Gyeongsang National University, Jinju, 660-701, Korea. ${ }^{2}$ Division of Science and Technology, U, rsity of Education, Township, Lahore, Pakistan. ${ }^{3}$ Department of Mathematics, Dong-A University, Busan, 604-714 rea.

\section{Ar knowledg ints}

tudy was s, pported by research funds from Dong-A University.

Receivt 9 September 2015 Accepted: 24 January 2016 Published online: 10 February 2016

\section{Re rences}

Stokes, GG: On the Effect of the Rotation of Cylinders and Spheres About Their Axes in Increasing the Logarithmic Decrement of the Arc of Vibration. Cambridge University Press, Cambridge (1886)

2. Casarella, MJ, Laura, PA: Drag on oscillating rod with longitudinal and torsional motion. J. Hydronaut. 3, 180-183 (1969)

3. Rajagopal, KR: Longitudinal and torsional oscillations of a rod in a non-Newtonian fluid. Acta Mech. 49, 281-285 (1983)

4. Rajagopal, KR, Bhatnagar, RK: Exact solutions for some simple flows of an Oldroyd-B fluid. Acta Mech. 113, 233-239 (1995)

5. Erdogan, ME: A note on an unsteady flow of a viscous fluid due to an oscillating plane wall. Int. J. Non-Linear Mech. $35,1-6(2000)$

6. Fetecau, C, Vieru, D, Fetecau, C: A note on the second problem of Stokes for Newtonian fluids. Int. J. Non-Linear Mech. 43, 451-457 (2008)

7. Fetecau, C, Fetecau, C: Starting solutions for some unsteady unidirectional flows of a second grade fluid. Int. J. Eng. Sci. 43, 781-789 (2005)

8. Fetecau, C, Fetecau, C: Starting solutions for the motion of a second grade fluid due to longitudinal and torsional oscillations of a circular cylinder. Int. J. Eng. Sci. 44, 788-796 (2006) 
9. Vieru, D, Akhtar, W, Fetecau, C, Fetecau, C: Starting solutions for the oscillating motion of a Maxwell fluid in cylindrical domains. Meccanica 42, 573-583 (2007)

10. Hayat, T, Siddiqui, AM, Asghar, S: Some simple flows of an Oldroyd-B fluid. Int. J. Eng. Sci. 39, 135-147 (2001)

11. Hayat, T, Mumtaz, S, Ellahi, R: MHD unsteady flows due to non-coaxial rotations of a disk and a fluid at infinity. Acta Mech. Sin. 19, 235-240 (2003)

12. Hayat, T, Ellahi, R, Asghar, S, Siddiqui, AM: Flow induced by non-coaxial rotation of a porous disk executing non-torsional oscillating and second grade fluid rotating at infinity. Appl. Math. Model. 28, 591-605 (2004)

13. Hayat, T, Ellahi, R, Asghar, S: Unsteady periodic flows of a magnetohydrodynamic fluid due to non-coaxial rotations of a porous disk and fluid at infinity. Math. Comput. Model. 40, 173-179 (2004)

14. Aksel, N, Fetecau, C, Scholle, M: Starting solutions for some unsteady unidirectional flows of Oldroyd-B fluids. Z. Angew. Math. Phys. 57, 815-831 (2006)

15. Khan, M, Ellahi, R, Hayat, T: Exact solution for oscillating flows of generalized Oldroyd-B fluid through porous medium in a rotating frame. J. Porous Media 12, 777-788 (2009)

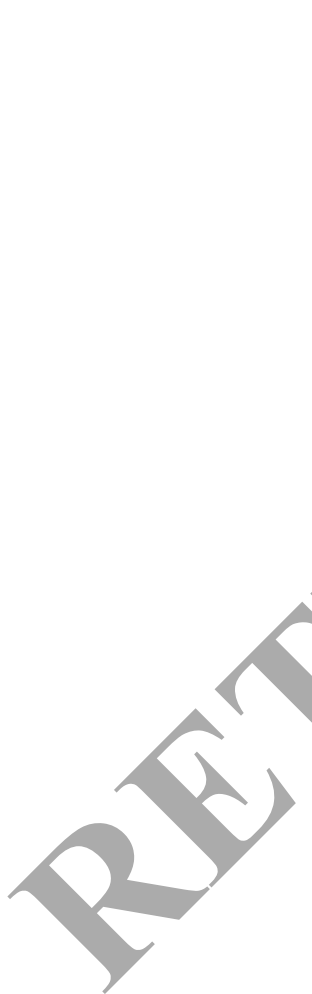

\section{Submit your manuscript to a SpringerOpen ${ }^{\circ}$ journal and benefit from:}

- Convenient online submission

- Rigorous peer review

- Immediate publication on acceptance

- Open access: articles freely available online

- High visibility within the field

- Retaining the copyright to your article 